\title{
Microflora of Patients with Stool Abnormality
}

\author{
Takashi Shimoyama, ${ }^{1 *}$ Shinji Hori, ${ }^{1}$ Kazutami Tamura, ${ }^{1}$ \\ Makoto Yamamura, ${ }^{1}$ Mamoru TANAKA ${ }^{2}$ and Kikuo YAmazaki ${ }^{2}$ \\ The 4th Department of Internal Medicine, Hyogo College of Medicine, 1-1, Mukogawa-cho, \\ Nishinomiya 663, 1 and Research Laboratory of Miyarisan Pharmaceutical Co., Ltd., \\ Togura-machi, Hanishina-gun, Nagano 389-082 \\ (Received 28 January, 1984)
}

Key words: Diarrhea; constipation; ulcerative colitis; abnormal intestinal flora; Bifidobacterium breve

Clinical symptoms such as abnormalities of stool habit, diarrhea and constipation are commonly experienced in an outpatient clinic, however, only a few studies have been reported on the intestinal microflora of outpatients, with the exception of patients with infectious diarrhea. Alterations of the intestinal microflora probably reflect the physiological state of the patient, and fermentative reactions due to microorganisms present in the gut may possibly induce diarrhea. Since 1970, bacteriological techniques for cultivating strictly obligate anaerobes have been vigorously developed and many studies on the intestinal microflora of patients with clinical problems have been reported in Japan. In the present paper, we report changes in the intestinal microflora of patients with acute diarrhea, maldigestion and malabsorption and with ulcerative colitis and also the effects of Bifidobacterium breve with transgalactosylated oligosaccharide on patients with chronic constipation. In this study the microbiological methods used were those of Mitsuoka (2), and the numbers of various bacteria were expressed as the $\log _{10}$ of the mean viable counts per gram of wet feces or per milliliter of intestinal contents. Detectable bacterial counts were more than 200 per gram of wet feces or per milliliter of intestinal contents.

\section{Fecal Microflora of Patients with Acute Diarrhea}

Fecal bacterial counts of 14 patients with acute diarrhea are shown in Table 1. These patients passed stools more than 4 times a day and most of them had watery diarrhea, abdominal pain or abdominal discomfort. Pathogenic organisms such as Salmonella spp., Campylobacter spp. and Shigella spp. were not isolated in these patients and diarrhea was probably due to viral infection. None of the patients had diarrhea beyond 2 weeks. In the feces of these patients compared with those of healthy subjects, the total number of obligate anaerobes was significantly decreased $(p<0.01)$, while the total number of facultative anaerobes showed a tendency to increase although there was no significant difference. Bifidobacteria were not isolated in 3 out of 14 patients and even in the detectable cases the bacterial counts were reduced in some cases. As a rule, bifidobacteria showed a tendency to decrease in patients having severe watery diarrhea. Bacteroidaceae were isolated in all of the patients and the bacterial counts were significantly decreased although they remained predominant in the fecal microflora $(p<0.05)$. Microaerophilic lactobacilli were significantly decreased $(p<0.05)$.

These findings suggest that it would be helpful to administer preparations of bifidobacteria or lactobacilli to patients with acute diarrhea in order to improve the abnormal state of the intestinal microflora.

The alterations in the fecal microflora of patients with diarrhea due to administration of antibiotics or anti-cancer drugs, specifically 5-FU derivatives, were more remarkable. 
Table 1. Fecal flora of patients with acute diarrhea

\begin{tabular}{|c|c|c|}
\hline Bacterial group & $\begin{array}{l}\text { Acute diarrhea } \\
\text { (14 cases) }\end{array}$ & $\begin{array}{c}\text { Healthy control } \\
\text { (37 cases) }\end{array}$ \\
\hline Obligate anaerobes & $10.4 \pm 0.4 * *(14)^{a}$ & $10.8 \pm 0.4(37)$ \\
\hline Bifidobacterium & $9.3 \pm 0.7 * \quad(11)$ & $9.8 \pm 0.7(37)$ \\
\hline Bacteroidaceae & $10.0 \pm 0.5^{*} \quad(14)$ & $10.4 \pm 0.5(37)$ \\
\hline Clostridium & $8.9 \pm 0.9$ & $8.7 \pm 1.3(32)$ \\
\hline Facultative anaerobes & $8.6 \pm 0.9$ & $8.1 \pm 0.9(37)$ \\
\hline Enterobacteriaceae & $7.9 \pm 1.3$ & $7.7 \pm 1.1(37)$ \\
\hline Streptococcus & $7.8 \pm 1.1$ & $7.3 \pm 1.0(37)$ \\
\hline Micrococcaceae & $4.0 \pm 1.2$ & $3.4 \pm 0.9(26)$ \\
\hline Lactobacillus & $5.4 \pm 1.4 * \quad(11)$ & $6.5 \pm 1.5(35)$ \\
\hline
\end{tabular}

a ( ): No. of subjects in whom the bacterial groups were present.

* Significantly different from the healthy control group $(* p<0.05, * * p<0.01)$.

In patients with bloody diarrhea due to administration of amoxicillin, Klebsiella oxytoca were isolated in some cases. The numbers of bifidobacteria and lactobacilli were markedly reduced. Similarly in cases with lincomycin, the numbers of bifidobacteria and lactobacilli were reduced. In the cases with cephalexin, the numbers of Micrococcaceae and streptococci were reduced. These findings suggest that carbohydrateutilizing bacterial groups such as bifidobacteria, lactobacilli or streptococci tend to be markedly reduced in patients with diarrhea due to administration of antibiotics. Consequently, it seems that administration of peroral drugs containing such bacteria is necessary when treating with antibiotics.

\section{Intestinal Microflora of Patients with Maldigestion and Malabsorption}

1) Fecal microflora of patients with chronic pancreatitis: In patients with chronic pancreatitis, maldigestion due to exocrine insufficiency of the pancreas results stool abnormality. The fecal bacterial counts of 22 patients with chronic pancreatitis and loose stools are shown in Table 2. Total obligate anaerobes were significantly decreased $(p<$ $0.05)$. Although bifidobacteria were isolated in 21 out of 22 patients, Bacteroidaceae and clostridia as well as bifidobacteria were significantly decreased $(p<0.05)$. There were no significant changes in the bacterial groups of facultative anaerobes, however, strepto- cocci showed a tendency to increase.

2) Fecal microflora of postgastrectomy patients: Approximately $10 \%$ of postgastrectomy patients have loose stools or diarrhea due to complicated maldigestion. Patients with gastrectomy (Billroth I; gastroduodenostomy) were divided into two subgroups according to stool condition: 12 cases with loose stools, 16 cases with formed stools. In both groups (Table 3, left half), bifidobacteria were significantly decreased while total facultative anaerobes, streptococci and lactobacilli, were significantly increased $(p<0.05)$. In the formed-stool group, clostridia were also significantly decreased $(p<0.05)$. The increase of streptococci was more remarkable in patients with loose stools than with formed stools. Another group of patients with gastrectomy (Billroth II, gastro-or esophagojejunostomy) were similarly divided into two subgroups: 13 cases with loose stools and 33 cases with formed stools. In both subgroups (Table 3, right half), bifidobacteria and clostridia were significantly decreased while total facultative anaerobes and Enterobacteriaceae were significantly increased $(p<0.05)$. Bifidobacteria were undetectable in 2 of 13 patients with loose stools and in 2 of 33 patients with formed stools. Streptococci and lactobacilli were significantly increased in patients with formed stools $(p<0.05)$.

3) Microflora in upper small intestinal contents of postgastrectomy patients: Studies on the human small intestine are complicated 
Table 2. Fecal flora of patients with chronic pancreatitis

\begin{tabular}{ccc}
\hline Bacterial group & $\begin{array}{c}\text { Chronic pancreatitis } \\
(22 \text { cases })\end{array}$ & $\begin{array}{c}\text { Healthy control } \\
\text { (37 cases) }\end{array}$ \\
\hline Obligate anaerobes & $10.4 \pm 0.5^{*}(22)^{a}$ & $10.8 \pm 0.4(37)$ \\
Bifidobacterium & $9.4 \pm 1.1^{*}(21)$ & $9.8 \pm 0.7(37)$ \\
Bacteroidaceae & $10.1 \pm 0.5^{*}(22)$ & $10.4 \pm 0.5(37)$ \\
Clostridium & $7.8 \pm 1.9^{*}(22)$ & $8.7 \pm 1.3(32)$ \\
Facultative anaerobes & $8.5 \pm 0.8(22)$ & $8.1 \pm 0.9(37)$ \\
Enterobacteriaceae & $7.7 \pm 1.4(22)$ & $7.7 \pm 1.1(37)$ \\
Streptococcus & $7.8 \pm 1.3(22)$ & $7.3 \pm 1.0(37)$ \\
Lactobacillus & $6.5 \pm 1.7(21)$ & $6.5 \pm 1.5(35)$ \\
\hline
\end{tabular}

a ( ): No. of subjects in whom the bacterial groups were present.

* Significantly different from the healthy control group $(p<0.05)$.

Table 3. Fecal flora of postgastrectomy patients

\begin{tabular}{|c|c|c|c|c|}
\hline \multirow{2}{*}{ Bacteria group } & \multicolumn{2}{|c|}{ Gastroduodenostomy } & \multicolumn{2}{|c|}{ Gastro (esophago) jejunostomy } \\
\hline & $\begin{array}{l}\text { Loose stool } \\
\text { (12 cases) }\end{array}$ & $\begin{array}{l}\text { Formed stool } \\
\text { (16 cases) }\end{array}$ & $\begin{array}{l}\text { Loose stool } \\
\text { (13 cases) }\end{array}$ & $\begin{array}{l}\text { Formed stool } \\
\text { (33 cases) }\end{array}$ \\
\hline Obligate anaerobes & $10.6 \pm 0.4 \quad(12)^{a}$ & $10.7 \pm 0.4 \quad(16)$ & $10.6 \pm 0.3 \quad(13)$ & $10.7 \pm 0.3$ \\
\hline Bifidobacterium & $9.3 \pm 0.6^{*}(11)$ & $9.1 \pm 0.8 *(15)$ & $9.2 \pm 0.6 *(11)$ & $9.2 \pm 1.2 *(31)$ \\
\hline Bacteroidaceae & $10.3 \pm 0.4 \quad(12)$ & $10.4 \pm 0.4 \quad(16)$ & $10.3 \pm 0.4 \quad(13)$ & $10.4 \pm 0.3$ \\
\hline Clostridium & $7.8 \pm 1.6 \quad(12)$ & $6.7 \pm 2.3^{*}(14)$ & $7.7 \pm 1.8 *(13)$ & $7.5 \pm 1.6^{*}(33)$ \\
\hline Facultative anaerobes & $8.7 \pm 0.7 *(12)$ & $8.7 \pm 0.6^{*}(16)$ & $8.9 \pm 1.0 *(13)$ & $9.1 \pm 0.6^{*}(33)$ \\
\hline Enterobacteriaceae & $7.8 \pm 1.2 \quad(12)$ & $8.2 \pm 0.9 \quad(16)$ & $8.4 \pm 0.9 *(13)$ & $8.6 \pm 0.6 *(33)$ \\
\hline Streptococcus & $8.5 \pm 0.7 *(12)$ & $8.1 \pm 1.2 *(16)$ & $8.4 \pm 1.5 \quad(13)$ & $8.7 \pm 0.9 *(33)$ \\
\hline Lactobacillus & $7.6 \pm 1.3 *(12)$ & $7.8 \pm 0.9 *(16)$ & $7.5 \pm 1.8 \quad(12)$ & $7.6 \pm 1.5 *(33)$ \\
\hline
\end{tabular}

by the problem of obtaining specimens from locations other than the mouth or anus. We devised a method of collecting small intestinal contents under physiological conditions using a free intestinal capsule which could be swallowed by the subject and worked by remote control when in the small intestine (1) and harvesting; the capsule was subsequently retrieved from the feces. We collected upper small intestinal contents using either the intestinal capsule or an endoscope. The concentrations of total obligate and facultative anaerobes were $10^{5}-10^{6}$ organisms $/ \mathrm{ml}$, respectively, in upper small intestinal contents in $80 \%$ of the healthy controls. Of obligate anaerobes Bacteroidaceae predominated, while in facultative anaerobes Enterobacteriaceae and lactobacilli were detectable in approximately $60 \%$ of cases and streptococci and yeasts in $40-50 \%$ of cases (Table 4 right).
In this study steatorrhea was defined by the criteria that the excretion rate of fecal fat is above $7 \%$ under loading of $50-60 \mathrm{~g}$ dietary fat. The microflora in the upper small intestinal contents of postgastrectomy patients either with or without steatorrhea were compared with those of healthy controls. In both postgastrectomy groups, the frequencies of occurrence of bifidobacteria, eubacteria and streptococci were markedly increased, and those of other bacterial groups were also increased. The bacterial counts were significantly increased in total obligate anaerobes, eubacteria, Bacteroidaceae, total facultative anaerobes, Enterobacteriaceae and streptococci $(p<0.05)$. Bacteroidaceae and eubacteria were significantly increased in patients with steatorrhea compared with patients without steatorrhea $(p<0.05)$.

According to stool condition (Table 5), total obligate anaerobes and Bacteroidaceae 
Table 4. Microflora in upper small intestinal contents of postgastrectomy patients with or without steatorrhea

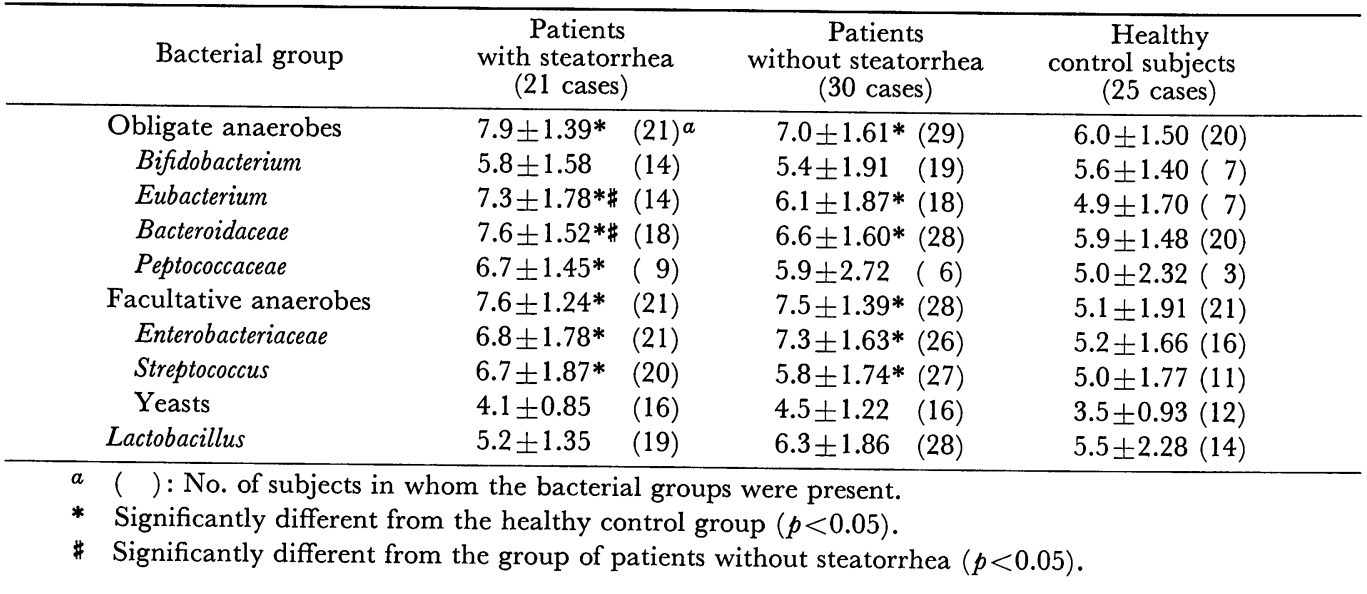

Table 5. Microflora in upper small intestinal contents of postagastrectomy patients

\begin{tabular}{|c|c|c|c|c|}
\hline \multirow{3}{*}{$\begin{array}{l}\text { Bacterial group } \\
\text { Obligate anaerobes }\end{array}$} & \multicolumn{2}{|c|}{ Gastroduodenostomy } & \multicolumn{2}{|c|}{ Gastro (esophago) jejunostomy } \\
\hline & \multirow{2}{*}{$\begin{array}{c}\begin{array}{c}\text { Loose stool } \\
(10 \text { cases })\end{array} \\
8.5 \pm 1.6^{*}(9)^{a}\end{array}$} & $\begin{array}{c}\text { Formed stool } \\
(12 \text { cases })\end{array}$ & $\begin{array}{l}\text { Loose stool } \\
\text { (21 cases) }\end{array}$ & $\begin{array}{l}\text { Formed stool } \\
\text { (14 cases) }\end{array}$ \\
\hline & & $6.5 \pm 1.4 \quad(12)$ & $8.4 \pm 1.4^{*}(21)$ & $6.8 \pm 2.0 \quad(13)$ \\
\hline Bifidobacterium & $6.9 \pm 2.3 \quad(5)$ & $5.3 \pm 1.3 \quad(2)$ & $6.7 \pm 1.9 \quad(12)$ & $5.4 \pm 2.4 \quad(9)$ \\
\hline Bacteroidaceae & $7.5 \pm 2.2 *(9)$ & $6.5 \pm 1.4 \quad(12)$ & $7.9 \pm 1.8 *(20)$ & $6.5 \pm 1.9 \quad(12)$ \\
\hline Clostridium & $5.2 \pm 2.3$ & $4.1 \pm 1.6 \quad(4)$ & $5.6 \pm 2.2 \quad(8)$ & $3.9 \pm 1.1 \quad(6)$ \\
\hline Facultative anaerobes & $7.0 \pm 1.3 *(9)$ & $7.4 \pm 1.1 \quad(11)$ & $8.1 \pm 1.3 *(21)$ & $7.5 \pm 1.7 *(13)$ \\
\hline Enterobacteriaceae & $6.5 \pm 1.3$ & $7.0 \pm 1.5^{*}(11)$ & $6.7 \pm 2.0 *(20)$ & $7.5 \pm 2.0 *(10)$ \\
\hline Streptococcus & $5.9 \pm 1.9$ & $5.7 \pm 1.4 \quad(11)$ & $7.7 \pm 1.4 \quad(21)$ & $4.3 \pm 1.6 \quad(10)$ \\
\hline Lactobacillus & $6.8 \pm 1.3 \quad(7)$ & $5.6 \pm 1.6 \quad(7)$ & $5.4 \pm 1.5$ & $6.0 \pm 2.1 \quad(14)$ \\
\hline
\end{tabular}

were significantly increased in patients with loose stools $(p<0.05)$. In these patients, total facultative anaerobes were also significantly increased $(p<0.05)$ and this tendency was more marked in patients with Billroth II gastrectomy than in those with Billroth I gastrectomy. Especially in patients with Billroth I gastrectomy having loose stools, total facultative anaerobes were markedly increased and the frequency of occurrence of streptococci was also high.

As mentioned above, the frequency of occurrence and the bacterial counts in the upper small intestinal contents of postgastrectomy patients were increased. In patients with loose stools, Bacteroidaceae and total obligate anaerobes were increased, suggesting the presence of the enforced fermentative reaction in the small intestine. The concentrations of Bacteroidaceae and eubacteria in the upper small intestinal contents were significantly increased compared with those of the patients without steatorrhea $(p<0.05)$. Forty-seven per cent of the strains of Bacteroidaceae were demonstrated to have the capability of taurocholate deconjugation, with $53 \%$ having the capability of glycocholate deconjugation. Deconjugation of conjugated bile acids by bacteria present in the upper small intestine impairs micellar phase formation which is a prerequisite of fat digestion and may result in steatorrhea. Therefore, the increase of Bacteroidaceae could be one of the causative 


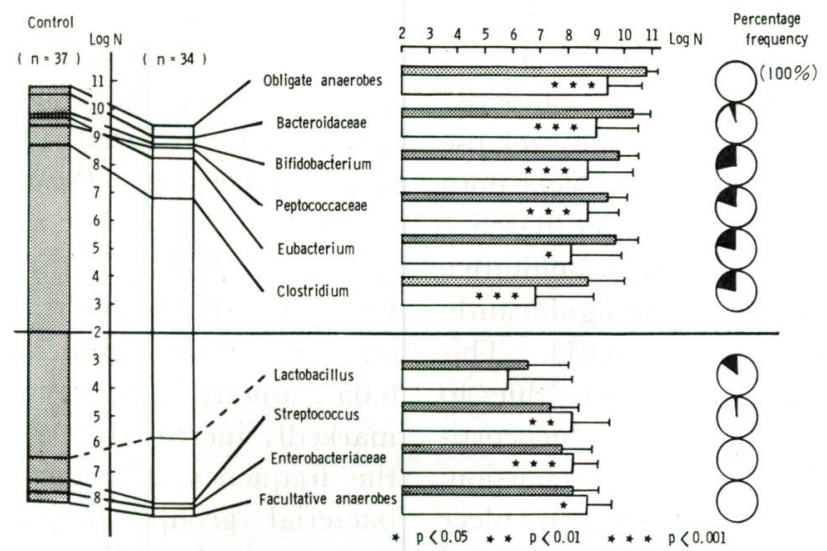

Fig. 1. Fecal bacterial counts of patients with ulcerative colitis (loose stool and diarrhea with blood).

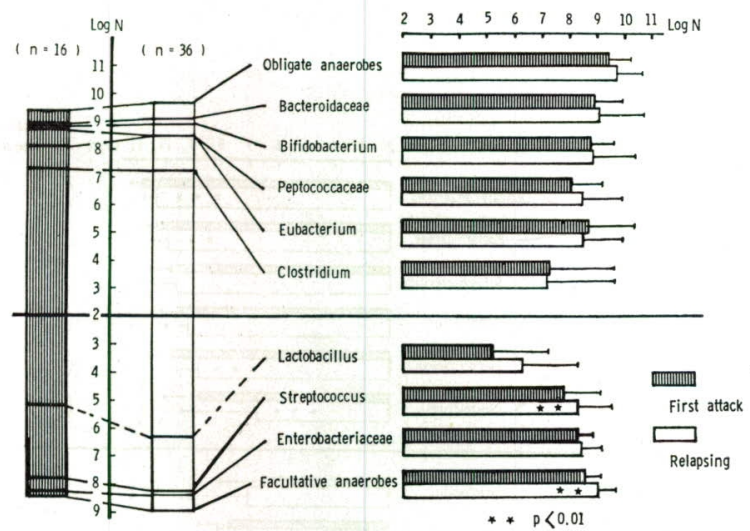

Fig. 2. Fecal bacterial counts of patients with ulcerative colitis (correlation with clinical course in active stage).

factors of steatorrhea.

\section{Fecal Microflora of Patients with Ulcera- tive Colitis}

Ulcerative colitis is a chronic disease of unknown etiology characterized by inflammation of the mucosa and submucosa of the large intestine. The most common symptom is rectal bleeding and the patient is usually in the second or third decade of life at the onset of symptoms.

The fecal microflora of active ulcerative colitis patients with bloody diarrhea is illustrated in Fig. 1. In obligate anaerobes, total counts and individual bacterial groups were significantly decreased. On the other hand, total facultative anaerobes, Enterobacteriaceae and streptococci were significantly increased. Of Enterobacteriaceae, Escherichia coli were decreased or undetectable in many cases and other genera of Enterobacteriaceae, especially Proteus, became predominant. In oligate anaerobes, total bacterial counts were decreased and in addition major bacterial groups were undetectable. This tendency was the most remarkable in bifidobacteria, which were undetectable in 35\% of patients. Peptococcaceae and eubacteria were both undetectable in $21 \%$ of patients. In patients with severe bloody diarrhea, Bacteroidaceae were undetectable $(6 \%)$. Lactobacilli were undetectable in $18 \%$ of cases.

Ulcerative colitis is mainly treated with 
glucocorticoids and Salazopyrin, relapse and remission often being repeated in this disease. The fecal flora were compared between untreated first attack and relapse cases since drugs might affect the fecal flora (Fig. 2). There was no significant difference in obligate anaerobes. Total facultative anaerobes and streptococci were significantly increased in relapse cases $(p<0.01)$. This increase of streptococci might be due to treatment with Salazopyrin or glucocorticoids. In ulcerative colitis in remission, endoscopical findings such as erosion, ulcer and bleeding of the mucosa were not observed at all. The fecal bacterial counts of patients with ulcerative colitis in remission are shown in Fig. 3. Despite the fact that most patients passed formed stools and bowel movements occurred once or twice per day, the number of total obligate anaerobes was significantly reduced $(p<0.001)$. Of obligate anaerobes Peptococcaceae, clostridia and Bacteroidaceae were significantly reduced $(p<0.001, p<0.001, p<0.01$, respectively). On the other hand, total facultative anaerobes were significantly increased $(p<$ $0.05)$, among them streptococci being most markedly increased $(p<0.001)$. As regards the frequency of occurrence of individual bacterial groups, bifidobacteria, Peptococcaceae and clostridia were undetectable in some cases. Lactobacilli were undetectable in $16 \%$ of the cases even in the remission stage.

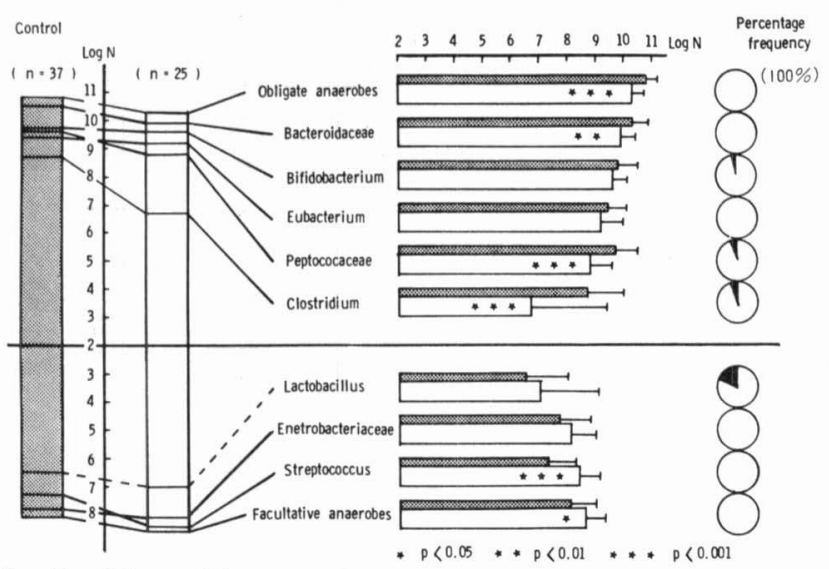

Fig. 3. Fecal bacterial counts of patients with ulcerative colitis in remission.

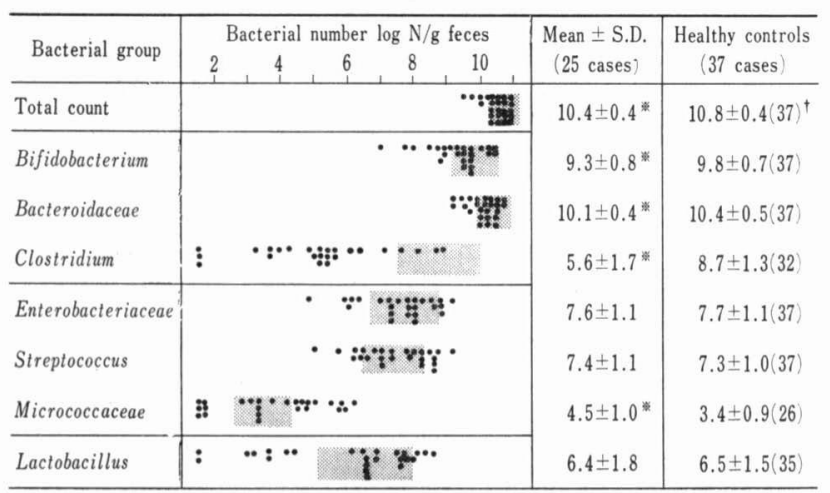

$\dagger$ Figures in parentheses indicate numbers of subjects in whom the bacterial groups were present.

* Significantly different from the healthy control group $(p<0.05)$. $\square$ : normal range.

Fig. 4. Fecal flora of patients with chronic constipation. 
Table 6. Clinical effects of BBG with TOS on constipated patients

\begin{tabular}{|c|c|c|c|}
\hline Disease & No. of cases & No. of effective cases & $\%$ Efficacy \\
\hline Cerebrovascular diseases & $6(1)^{a}$ & $3(1)$ & \multirow{2}{*}{63} \\
\hline Disorders of the spinal cord & $2(2)$ & $2(2)$ & \\
\hline Diabetes mellitus, cholelithiasis & $8(4)$ & $5(3)$ & 63 \\
\hline Hepatitis, cirrhosis of the liver & $4(1)$ & $2(0)$ & 50 \\
\hline Intraperitoneal adhesion & $4(4)$ & $2(2)$ & 50 \\
\hline Sigma elongation & $3(2)$ & $2(1)$ & \multirow{2}{*}{63} \\
\hline Irritable bowel syndrome & $5(3)$ & $3(2)$ & \\
\hline Miscellaneous & & & \\
\hline Multiple gastric ulcer & $1(1)$ & 0 & \\
\hline Chronic hepatitis & $1(0)$ & 0 & \\
\hline Total & $34(19)$ & $19(11)$ & 56 \\
\hline
\end{tabular}

a Figures in parentheses indicate numbers of patients treated with laxatives or enemas.

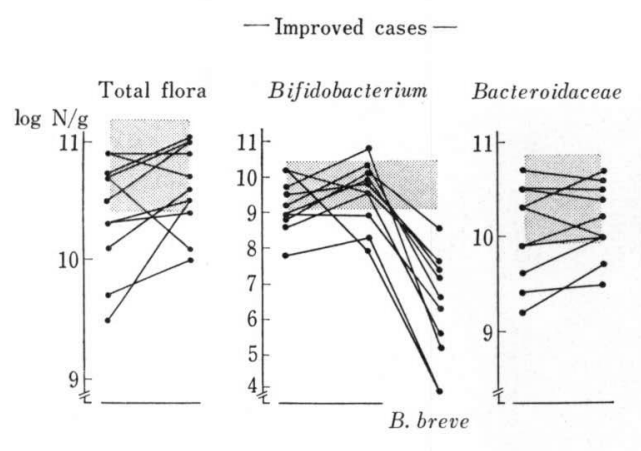

Non-effective or slightly improved cases -

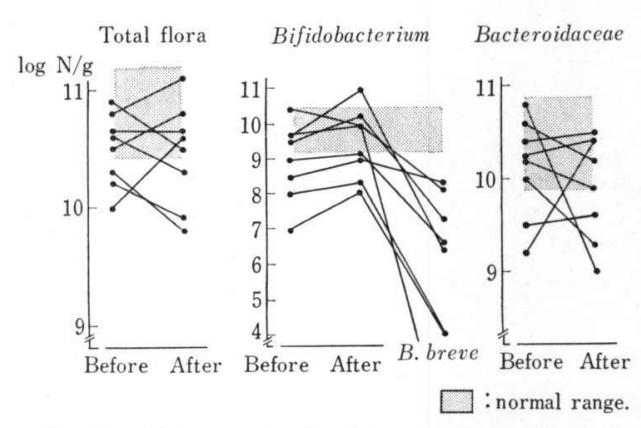

Fig. 5. Changes in fecal flora of constipated patients after administering BBG+TOS.

As mentioned above, in ulcerative colitis, regardless of the stage of the disease, total obligate anaerobes were significantly decreased while total facultative anaerobes were significantly increased. This tendency was also observed in formed stools. In addition, bifidobacteria, Peptococcaceae or lactobacilli were undetectable in many cases and $E$. coli were remarkably reduced or undetectable in active ulcerative colitis patients. These alterations in the fecal flora suggest the presence of abnormal fermentation reactions occurring in the gut of ulcerative colitis patients. This was supported by the analysis of short-chain fatty acids in the feces of ulcerative colitis patients. Consequently, it seems that it is necessary to increase bifidobacteria or lactobacilli when treating ulcerative colitis patients.

\section{Fecal Flora of Constipated Patients and Clinical Effects of BBG with TOS}

The fecal bacterial counts of patients with chronic constipation are shown in Fig. 4. Obligate anaerobes such as bifidobacteria, Bacteroidaceae and clostridia were significantly reduced in constipated patients $(p<0.05)$. There was no consistent tendency in facultative anaerobes and lactobacilli.

The clinical effects of BBG (Bifidobacterium breve) with TOS (transgalactosylated oligosaccharide) on constipated patients are shown in Table 6. BBG (6 g/day: $1-3 \times$ $\left.10^{9} / \mathrm{g}\right)$ together with TOS (15 g/day) was administered to patients with chronic constipation due to various diseases, and symptoms improved in 19 out of 34 patients (56\% efficacy). The changes in the fecal flora of constipated patients after administering BBG with TOS are shown in Fig. 5. In the improved cases, total bacterial count 
and bifidobacteria showed a tendency to increase, while in the non-effective or slightly improved cases only bifidobacteria showed a tendency to increase although this tendency was less marked than in the improved cases. The concentrations of Bifidobacterium breve were relatively low suggesting that the increase of bifidobacteria was mainly due to an increase of indigenous species of bifidobacteria.

\section{References}

(1) Hori, S., K. Asaka, K. Tamura, I. Ohama, and T. Shimoyama. 1982. Study on the microflora in the small intestine of healthy subjects using the intestinal capsules. Abstract Book of the World Congresses in Stockholm Sweden. p. 441.

(2) Mitsuoka, T., T. Sega, und S. Yamamoto. 1965. Eine verbesserte Methodik der qualitativen und quantitativen Analyse der Darmflora von Menschen und Tiere. Zbl. Bakt. Hyg. I. Orig. 195: 455-469. 